\title{
Comparative study of control strategies for oscillation damping in DC microgrids
}

\author{
Isaías Valente de Bessa * Renan Landau Paiva de Medeiros * \\ Iury Valente de Bessa* João Edgar Chaves Filho* \\ Florindo A.C. Ayres Junior* \\ * Faculdade de Tecnologia, Universidade Federal do Amazonas, AM \\ (e-mails: isaias.97.ib@gmail.com,renanlandau@ufam.edu.br, \\ iurybessa@ufam.edu.br, joaoedgarc@gmail.com, \\ florindoayres@ufam.edu.br).
}

\begin{abstract}
Nowadays, the use of converters is attracting increasing attention due to diversification of electric power sources. In particular, DC-DC converters are used to ensure the stability in the DC supply responsible for feeding a DC microgrid with constant voltage. Thus, classical and modern controls methodologies are used to ensure the stability of the feed system when connected to a constant power load (CPL). This paper compares control methodologies for pole allocation using Diophantine equation, root locus, linear-quadratic regulator and the Lyapunov equation by using performance indices as integral of square error (ISE), integral of square weighted by time (ISET) and integral of square of control (ISSC).
\end{abstract}

Keywords: DC-DC power converters; DC microgrid; CPL; controls methodologies; perfomance index.

\section{INTRODUCTION}

The demand for new energy sources results in the increasing use of converters in power systems whether by the need to convert the $\mathrm{AC}$ source from the grid into a DC level for the power supply of electrical devices or the conversion of $\mathrm{DC}$ voltage into $\mathrm{AC}$ voltage for the return to the electric grid. However, between steps AC-DC and $\mathrm{DC}-\mathrm{AC}$, the treatment of this voltage are done by $\mathrm{DC}$ DC converters capable of stabilizing the voltage from the AC-DC converter (Elsayed et al., 2015).

Due to the demand for DC-DC converters, its modeling has been extensively investigated in the literature, (Erickson and Maksimović, 2004), as well as control strategies for voltage regulation. In particular, in (Poodeh et al., 2007) is proposed a control strategy for buck converter using Fuzzy logic. Modern control techniques have also been used for regulation of buck converters, as in (Maccari et al., 2016) that uses LQR optimal control, where the buck is used in the auxiliary power system of an electric vehicle.

The modeling and control of DC-DC converters allow the construction of DC microgrids. Microgrids are composed of feeders that must maintain a constant voltage of DC bus to which are connected DC loads as in (Marcillo et al., 2019; Singh et al., 2017). The behavior of the constant power loads (CPL) in the connection with the DC bus is studied in (Mosskull, 2018), where it is observed oscillations in the feeder due to the constant consumption of power of the CPL that causes a variation of current and voltage for the maintenance of the constant power. These variations result in the occurrence of a negative resistance in which its effects are observed in the form of oscillations in the DC bus (Marcillo et al., 2019; Singh et al., 2017).

Ensuring the stability of the microgrid is fundamental to the proper functioning of the system and in (Riccobono and Santi, 2014) it is shown criteria that guarantee the stability of the system among which stands out the criterion of
Middlebrook that ensure the DC microgrid stability if the output feeder impedance is less than the input impedance of CPL. The mitigation of CPL oscillations is already investigated in (Mosskull, 2018) that shows the relationship between good damping and small power variations, and in (Marcillo et al., 2019), that uses robust control strategies to reduce the oscillation effects.

This paper proposes a DC microgrid model where the feeder is represented by a boost converter while the CPL is designed through a buck converter. Through the connection between the two systems, it is sought to investigate control strategies for the regulation of the feeder capable of mitigating the oscillations arising from the connection to the CPL. Among the proposed structures are the output feedback structures using PID controllers designed by pole placement metrics using the Diophantine equation (DE) and graphical use of the root locus (RL) for the determination of the region of stability and performance of the system to be controlled. State feedback structures are also considered in the analysis, where the determination of controllers gain is performed using the linear-quadratic regulator (LQR) and the solution of the Lyapunov equation. Finally, an analysis of the performance index of each proposed method was performed, observing the controllers ability to mitigate the oscillations from the CPL.

The remaining of this paper is divided as follows: In Section 2 the modeling of the converters constituting the microgrid is discussed. In Section 3 a revision of control strategies to be used to stabilize the feeder in connection with the CPL is carried out. Section 4 shows the experimental procedures and the test performed to verify the performance of the feeder controllers. In Section 5 the controllers performance analysis is evaluated through performance index graphs. Finally, in Section 6 the final considerations are made about the proposal of mitigation of oscillations in the feeder, observing the results analyzed in Section 5. 


\section{MODELING OF DC MICROGRIDS WITH CPL}

\subsection{Modeling of the boost converter operating as a feeder}

The boost converter showed in Fig. 1a is responsible for stepping up an input voltage level $V_{i 1}$ to an output level $V_{o 1}$, according to the relation expressed by (1), for a duty cycle $D_{1}$ (Erickson and Maksimović, 2004).

$$
V_{o 1}=\frac{V_{i 1}}{1-D_{1}}
$$

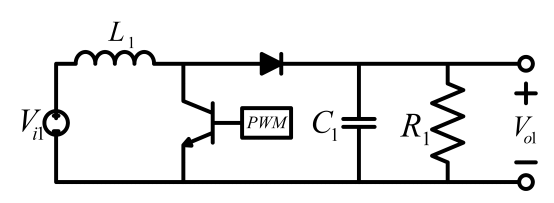

(a)

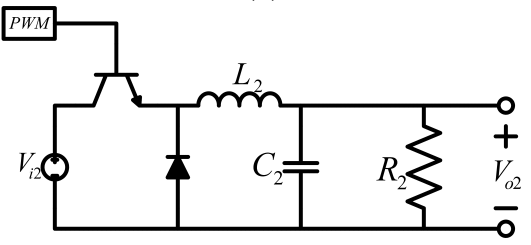

(b)

Fig. 1. (a) Boost converter (b) Buck converter.

The inductor $L_{1}$ is determined to ensure continuous conduction operation mode $\left(I_{L_{1}}>0\right)$ and for a signal PWM of frequency, $f_{1}$ is obtained the variation of current $\Delta I_{L_{1}}$ in the inductor and the voltage variation $\Delta V_{o 1}$ in the capacitor. These relations are described as follows (Erickson and Maksimović, 2004).

$$
\left\{\begin{array}{l}
L_{1}>\frac{R_{1} D_{1}\left(1-D_{1}\right)^{2}}{2 f_{1}} \\
\Delta I_{L_{1}}=\frac{D_{1} V_{i 1}}{L_{1} f_{1}} \\
\frac{\Delta V_{o 1}}{V_{o 1}}=\frac{D_{1}}{f_{1} R_{1} C_{1}}
\end{array}\right.
$$

The set of equations shown in (1) and (2) are derived from the analysis of the steady-state boost converter. The dynamic behavior is obtained through the circuit analysis of Fig. 1a in two moments: when the PWM signal is high level and low level, shown respectively in (3) and (4), as described in (Erickson and Maksimović, 2004).

$$
\begin{gathered}
\left\{\begin{array}{l}
\dot{I}_{L_{1}}=\frac{V_{i 1}}{L_{1}} \\
\dot{V}_{C_{1}}=-\frac{V_{C_{1}}}{R_{1} C_{1}}
\end{array}\right. \\
\left\{\begin{array}{l}
\dot{I}_{L_{1}}=-\frac{V_{C_{1}}}{L_{1}}+\frac{V_{i 1}}{L_{1}} \\
\dot{V}_{C_{1}}=\frac{I_{L_{1}}}{C_{1}}-\frac{V_{C_{1}}}{R_{1} C_{1}}
\end{array}\right.
\end{gathered}
$$

To obtain a representative model of the system in the two instants of operation, it is used the average space model (Erickson and Maksimović, 2004) shown in (5) where the output information is the voltage $V_{o 1}$ on resistor $R_{1}$.

$$
\left\{\begin{array}{l}
{\left[\begin{array}{l}
\dot{I}_{L_{1}} \\
\dot{V}_{C_{1}}
\end{array}\right]=\left[\begin{array}{cc}
0 & -\frac{1-D_{1}}{L_{1}} \\
\frac{1-D_{1}}{C_{1}} & -\frac{1}{R_{1} C_{1}}
\end{array}\right]\left[\begin{array}{l}
I_{L_{1}} \\
V_{C_{1}}
\end{array}\right]+\left[\begin{array}{c}
\frac{1}{L_{1}} \\
0
\end{array}\right]\left[V_{i 1}\right]} \\
V_{o 1}=V_{C_{1}}
\end{array}\right.
$$

For the feeder output regulation the value of the source $V_{i 1}$ is constant, so the control of the output signal is performed from the duty cycle $D_{1}$ of the circuit, consisting of a fixed part $D_{1}^{o}$ associated to the operating point of the circuit and another variable part $\Delta D_{1}$, as shown in (6).

$$
D_{1}(t)=D_{1}^{o}+\Delta D_{1}
$$

Thus, by linearizing the system around the operating point $\left(D_{1}^{o}, I_{L 1}^{o}, V_{C 1}^{o}\right)$, the state-space model presented in $(7)$ is obtained.

$$
\left\{\begin{array}{c}
{\left[\begin{array}{c}
\Delta \dot{I}_{L_{1}} \\
\Delta \dot{V}_{C_{1}}
\end{array}\right]=\left[\begin{array}{cc}
0 & -\frac{1-D_{1}^{o}}{L_{1}} \\
\frac{1-D_{1}^{o}}{C_{1}} & -\frac{1}{R_{1} C_{1}}
\end{array}\right]\left[\begin{array}{c}
\Delta I_{L_{1}} \\
\Delta V_{C_{1}}
\end{array}\right]+\left[\begin{array}{c}
\frac{V_{C_{1}}^{o}}{L_{1}} \\
-\frac{I_{L_{1}}^{b}}{C_{1}}
\end{array}\right]\left[\Delta D_{1}\right]} \\
\Delta V_{o 1}=\Delta V_{C_{1}}
\end{array}\right.
$$

where $V_{C_{1}}^{o}=\frac{V_{i 1}}{1-D_{1}^{o}}$ and $I_{L_{1}}^{o}=\frac{V_{i 1}}{R\left(1-D_{1}^{o}\right)^{2}}$ are respectively the voltage in the capacitor and the current in the inductor at the point of operation with duty cycle $D_{1}^{o}$ described as follows. Finally, based on (7), the following transfer function model can be obtained for the boost converter

$$
G(s)=\frac{\Delta V_{o 1}(s)}{\Delta D_{1}(s)}=\frac{-\frac{I_{L_{1}}^{o}}{C_{1}} s+\frac{\left(1-D_{1}^{o}\right) V_{C_{1}}^{o}}{L_{1} C_{1}}}{s^{2}+\frac{1}{R_{1} C_{1}} s+\frac{\left(1-D_{1}^{o}\right)^{2}}{L_{1} C_{1}}}
$$

\subsection{Modeling of the buck converter operating as CPL}

The buck converter is shown in Fig. 1b is responsible for step down a voltage level $V_{i 2}$ at the input to a voltage level $V_{o 2}$ at the output through the ratio shown in (9), where $D_{2}$ represents the duty cycle of the circuit operation (Erickson and Maksimović, 2004).

$$
V_{o 2}=D_{2} V_{i 2}
$$

The inductor $L_{2}$ must be such that its value guarantees the operation of the circuit in continuous conduction $\left(I_{L_{2}}>0\right)$ and for a signal PWM of frequency $f_{2}$ is can determine the variation of current $\Delta I_{L_{2}}$ in the inductor and the voltage variation $\Delta V_{o 2}$ in the output of the circuit. These relationships are shown in (10) and shown in more detail in (Erickson and Maksimović, 2004).

$$
\left\{\begin{array}{l}
L_{2}>\frac{R_{2}\left(1-D_{2}\right)}{2 f_{2}} \\
\Delta I_{L_{2}}=\frac{D_{2}\left(1-D_{2}\right) V_{i 2}}{L_{2} f_{2}} \\
\frac{\Delta V_{o 2}}{V_{o 2}}=\frac{1-D_{2}}{8 f_{2}{ }^{2} R_{2} C_{2}}
\end{array}\right.
$$

For this paper the buck is treated as CPL, so the output power of the converter is given by (11) using as the basis for power $D_{2}=1$.

$$
P_{o 2}=D_{2}^{2}
$$


By performing the same procedures to obtain an average space-state and linearization around the operating point $\left(D_{2}^{o}, I_{L_{2}}^{o}, V_{C_{2}}^{o}\right)$, the linearized model in (12) is given.

$$
\left\{\begin{array}{c}
\left.\left[\begin{array}{c}
\Delta \dot{I}_{L_{2}} \\
\Delta \dot{V}_{C_{2}}
\end{array}\right]=\left[\begin{array}{cc}
0 & -\frac{1}{L_{2}} \\
\frac{1}{C_{2}} & -\frac{1}{R_{2} C_{2}}
\end{array}\right] \underset{2 D_{2}^{o}}{ } \begin{array}{c}
\Delta I_{L_{2}} \\
\Delta V_{C_{2}}
\end{array}\right]+\left[\begin{array}{c}
\frac{V_{i 2}}{L_{2}} \\
0
\end{array}\right]\left[\Delta D_{2}\right] \\
\Delta P_{o 2}=\frac{2 V_{C_{2}}}{V_{i 2}}
\end{array}\right.
$$

With the state space linearized system in (12), the transfer function of the buck converter was determined for variations around the operating point in (13).

$$
H(s)=\frac{\Delta P_{o 2}(s)}{\Delta D_{2}(s)}=\frac{\frac{2 D_{2}^{o}}{L_{2} C_{2}}}{s^{2}+\frac{1}{R_{2} C_{2}} s+\frac{1}{L_{2} C_{2}}}
$$

\section{CONTROL STRATEGY FOR DC MICROGRIDS WITH CPL}

For the implementation of the microgrid, the voltage regulation from the feeder is fundamental. In this way, the investigation of control strategies becomes necessary. These include classical methods such as the DE and the use of the RL graphical tool in the use of output feedback structures shown in Fig. 2a, show in (Chen, 1999). The state feedback structure, shown in Fig. 2b, is also used for the implementation of the LQR control, (D'Azzo and Houpius, 1988) or through the solution of the LE, (Chen, 1999).
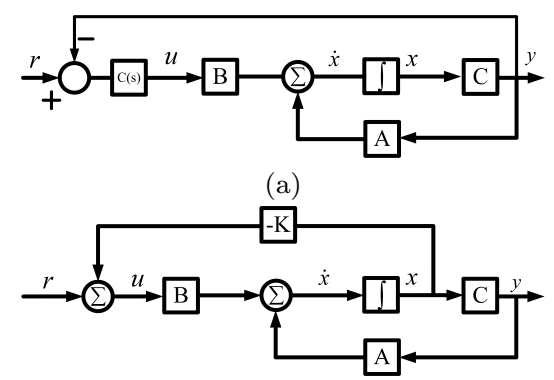

(b)

Fig. 2. (a) System with output feedback structure (b) System with state feedback structure.

\subsection{The Diophantine equation}

Consider the output feedback structure system shown in Fig. 2a. The open-loop system transfer function $G(s)=$ $\frac{B(s)}{A(s)}$ is calculated directly through (14).

$$
G(s)=C(s I-A)^{-1} B
$$

When performing the system output feedback, it will have a characteristic equation $\Delta_{c}(s)$, shown in (15), to represent its dynamic behavior, dependent on the designed $C(s)=$ $\frac{N(s)}{M(s)}$ controller.

$$
\Delta_{c}(s)=1+C(s) G(s)
$$

Give a polynomial $\Delta_{d}(s)$ desired for the closed-loop system. Comparing this polynomial to the characteristic polynomial presented in (15), has the Diophantine equation, presented in (16).

$$
M(s) A(s)+N(s) B(s)=\Delta_{d}(s)
$$

The solution of the polynomial equation proposed in (16), when possible, allows the determination of the parameters of the controller $C(s)$, by determining the coefficients of polynomials $M(s)$ and $N(s)$ (Chen, 1999).

\subsection{Region of system performance through root locus}

Another way to ensure the desired performance of a closedloop system given the transfer function $G(s)$ is through the use of the root locus tool, which given a variable parameter in controller $C(s)$, shows the behavior of the poles of the closed-loop system of the desired polynomial $\Delta_{d}(s)$, (D'Azzo and Houpius, 1988).

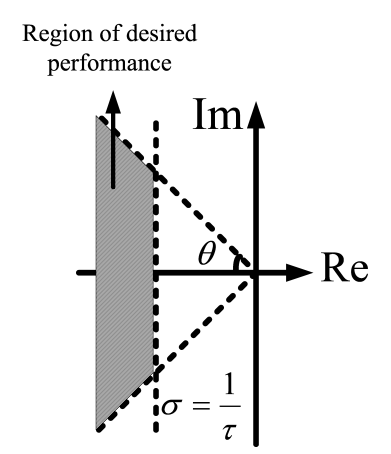

Fig. 3. Desired performance region on root locus

The desired polynomial is formed by design specifications such as settling time $t_{s s}$ and damping coefficient $\zeta$.

The settling time is related to the time constant $\tau \simeq \frac{t_{s s}}{5}$ of the system to be analyzed. Where the damping $\sigma$ of the system is related to the time constant as indicated in (17).

$$
\sigma=\frac{1}{\tau} \quad \zeta=\cos \theta
$$

The damping coefficient $\zeta$ is related to the angle $\theta$ that the line segment from the origin to the dominant pole realizes with the real axis, as indicated in (17). Thus, the desired performance region in the plane of the complex is determined, as indicated in Fig. 3, where the desired performance region is hatched.

\subsection{The linear-quadratic regulator}

The LQR is an optimal control methodology capable of allowing the designer choosing between minimizing energy or minimizing time, by developing the Quadratic Performance Index shown in (18), (D'Azzo and Houpius, 1988).

$$
I=\int_{t}\left(x^{T} Q x+u^{T} R u\right) d t
$$

For the use of the LQR methodology, the state feedback structure is used, as shown in Fig. 2b. The solution of the optimal control problem is to determine the gain value $K$ for the state feedback, utilizing the solution of the Riccati equation, see (D'Azzo and Houpius, 1988), shown in (19) resulting from the development of (18).

$$
P A+A^{T} P-P B R^{-1} B^{T} P=-Q
$$


where $\mathrm{Q}$ is symmetric semi-definite positive matrice and $R$ is a defined positive determined by the designer. The solution of (19) results in the definition of the matrice $P$. The values of the gains of the matrix $K$ are given by (20).

$$
K=R^{-1} B^{T} P
$$

The weighting of $Q$ and $R$ are related to the project's need to prioritize better performance or less control effort.

\subsection{Lyapunov equation}

Considering the system presented in the open-loop controlled Fig. 2b, the gain value $K$ is determined for the system to satisfy specifications by the solution of the Lyapunov equation shown in (21) (Chen, 1999).

$$
A T-T F=B K_{T}
$$

Where $F$ is a matrice whose eigenvalues are equal to the roots of the desired polynomial $\Delta_{d}(s)$. The matrice $K_{T}$ is arbitrarily chosen such that $F K_{T}$ is observable and the matrice $T$ is the solution of the Lyapunov equation. With the matrice $T$, the state feedback gain $K$ is determined through (22).

$$
K=K_{T} T^{-1}
$$

\section{METHODOLOGICAL PROCEDURES}

This paper proposes to evaluate the performance of the controllers proposed for the feeder for the voltage regulation in the DC bus of the microgrid proposed in Fig. 4. The design of the parameters of the microgrid as well as the gains of the controllers is discussed in this section besides the test performed for the validation and analysis of the performance of the controllers.

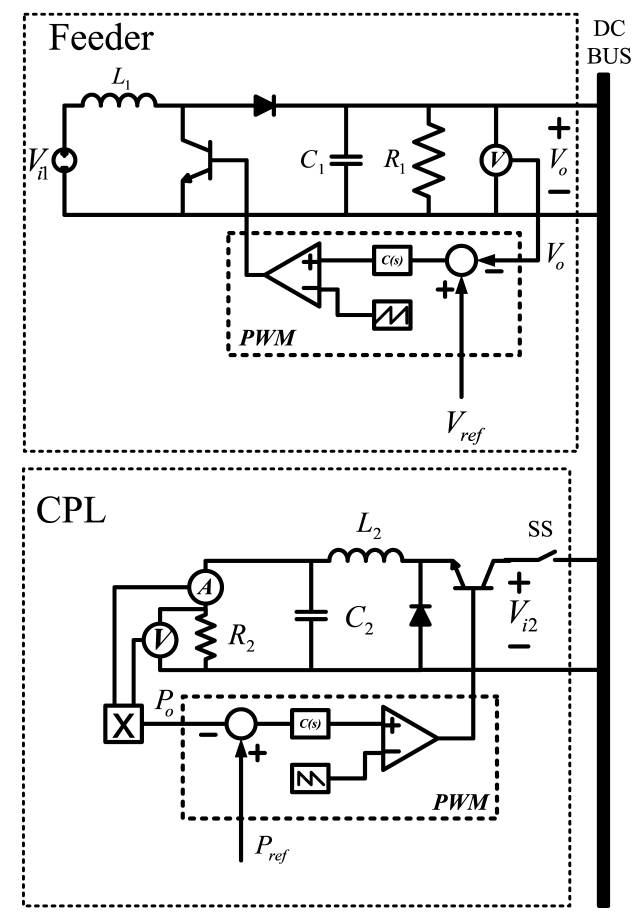

Fig. 4. Proposed DC microgrid.

\subsection{DC microgrid design}

The feeder is represented for a boost converter, and its project is based on a switching frequency $f_{1}=2 \mathrm{KHz}$, limited by $L_{1}=1 \mathrm{mH}$ inductor and an input voltage $V_{i 1}=12 \mathrm{~V}$. In this way, the resistance parameters $R_{1}$, capacitor $C_{1}$, duty cycle $D_{1}$ and output voltage $V_{o 1}$ were determined for an input current variation of $\Delta I_{L_{1}} \leq 2 \mathrm{~A}$ based on the set of equations shown in (2).

On the other hand, the design of the CPL, represented for a buck converter is based on a switching frequency $f_{2}=4 \mathrm{KHz}$, limited by $L_{2}=1 \mathrm{mH}$ inductor and an input voltage $V_{i 2}=18 \mathrm{~V}$. In this way, the resistance parameters $R_{2}$, capacitor $C_{2}$, Duty Cycle $D_{2}$ and output voltage $V_{o 2}$ are determined for an input current variation of $\Delta I_{L_{2}} \leq 1.5 \mathrm{~A}$ based on the set of equations shown in (10). Table 1 shows the computed parameters.

The rated power of the CPL is determined for $D_{2}=1$, where for an input voltage of $1 V_{i 2}=18 \mathrm{~V}$ and a load of $R_{2}=5 \Omega$. Thus, the rated power of the CPL is given by

\begin{tabular}{|c|c|c|c|}
\hline \multicolumn{4}{|c|}{ Boost feeder } \\
\hline Parameter & Symbol & Value & Unit \\
\hline Input voltage & $\overline{V_{i 1}}$ & 12.0 & $\bar{V}$ \\
\hline Duty cycle & $D_{1}$ & 0.33 & - \\
\hline Switching frequency & $f_{1}$ & 2.0 & $\mathrm{KHz}$ \\
\hline Load resistance & $R_{1}$ & 5.0 & $\Omega$ \\
\hline Inductor & $L_{1}$ & 1.0 & $\mathrm{mH}$ \\
\hline Capacitor & $C_{1}$ & 2.0 & $\mathrm{mF}$ \\
\hline Output voltage & $V_{o 1}$ & 18.0 & V \\
\hline \multicolumn{4}{|c|}{ Buck CPL } \\
\hline Parameter & Symbol & Value & Unit \\
\hline Input voltage & $V_{i 2}$ & 18.0 & $\mathrm{~V}$ \\
\hline Duty cycle & $D_{2}$ & 0.6 & - \\
\hline Switching frequency & $f_{2}$ & 4.0 & $\mathrm{KHz}$ \\
\hline Load resistance & $R_{2}$ & 5.0 & $\Omega$ \\
\hline Inductor & $L_{2}$ & 1.0 & $\mathrm{mH}$ \\
\hline Capacitor & $C_{2}$ & 2.0 & $\mathrm{mF}$ \\
\hline Output power & $P_{o 2}$ & 0.36 & $\mathrm{pu}$ \\
\hline
\end{tabular}
$P_{n}=\frac{V_{i}^{2}}{R_{2}}=64.8 \mathrm{~W}$.

Table 1. Microgrid parameters

The CPL switching frequency is twice as fast as the feeder. This way, the feeder becomes sensitive to instantaneous CPL power variations.

\subsection{Controller design}

After the determination of the parameters of the converters for the feeder and the CPL, the controller design is carried out. The controller structures used are shown in Fig. 5.

For the feeder and CPL controllers are designed to meet a settling time $t_{s s}$, an overshoot ovs and a system with steady-state error $e_{s s}$ at step with specifications shown in (23) and (24).

$$
\begin{gathered}
t_{s s}<0.02 s, \text { ovs }<10 \%, \quad e_{s s}=0 \\
\text { ovs }=e^{-\frac{\zeta \pi}{\sqrt{1-\zeta^{2}}}}, \quad t_{s s}=\frac{4.6}{\zeta \omega_{n}}
\end{gathered}
$$

For the proposed controller structure for the feeder, two auxiliary poles, $p_{1}$ and $p_{2}$, are used to ensure that the Diophantine equation is possible and determinable while for the proposed CPL structure an auxiliary pole $p_{3}$ is used. They are allocated so as not to interfere with the 
desired behavior of the systems. The relationships used are shown in (25).

$$
p_{1}=2 \zeta \omega_{n}, \quad p_{2}=2.5 \zeta \omega_{n}, \quad p_{3}=2 \zeta \omega_{n}
$$

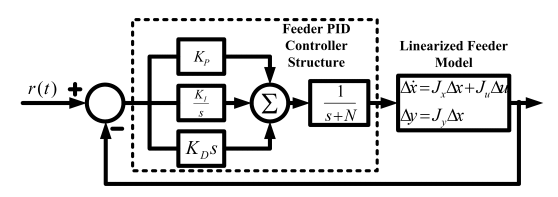

(a)

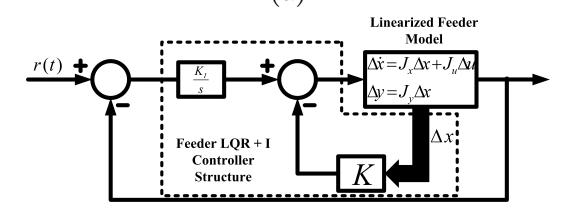

(b)

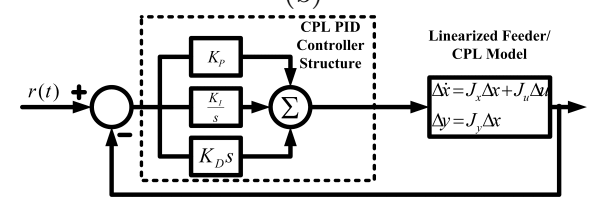

(c)

Fig. 5. Structure proposed for the design of the boost converter controller (a) with output feedback (b) with state feedback and (c) the proposed structure for the buck converter controller as CPL.

With the information obtained from the (24) and (25) relations, the desired polynomials for the feeder, $\Delta_{d f}(s)$ and CPL, $\Delta_{d c}(s)$ are constructed as $(26)$. The gains of the CPL controllers were calculated such that $K_{p}=-0.1679$, $K_{i}=127.659$ and $K_{d}=0.0015$.

$$
\left\{\begin{array}{l}
\Delta_{d f}(s)=\left(s^{2}+2 \zeta \omega_{n} s+\omega_{n}^{2}\right)\left(s+p_{1}\right)\left(s+p_{2}\right) \\
\Delta_{d c}(s)=\left(s^{2}+2 \zeta \omega_{n} s+\omega_{n}^{2}\right)\left(s+p_{3}\right)
\end{array}\right.
$$

The feeder controller is also designed using the root locus (RL). With the expected performance of the system presented in the system and with the help of the rltool() tool of MATLAB, the performance region is determined to place the poles, as indicated in Fig. 3.

The feeder controller is also designed using the LinearQuadratic Regulator (LQR) method using the structure shown in Fig. 5b. For the problem solving of the Riccati equation the matrices, $Q$ and $R$ are chosen as indicated in (27). Where the weighting of a value elevated to $R$ causes a penalty in the control action. The value of the integral gain is determined from the increased matrice of the system, to guarantee zero error to the steadystate step. The gain vector $K$ was calculated using the MATLAB $\operatorname{lqr}()$ command.

$$
Q=\left[\begin{array}{cc}
1 & 0 \\
0 & 10^{-6}
\end{array}\right], R=100
$$

Finally, the controller gains are projected using the Lyapunov equation (LE), whereas in the LQR structure, shown in Fig. 5b is used with an integrator at the input to ensure zero error to the step. The $F$ matrice, shown in (28), is constructed by determining the desired polynomial with the system specifications presented in (23). The MATLAB $\operatorname{lyap}()$ command is used for the determination of the gain vector $K$. The controller gains are summarized in Table 2.

$$
F=\left[\begin{array}{cc}
\zeta \omega_{n} & \omega_{n} \sqrt{1-\zeta^{2}} \\
-\omega_{n} \sqrt{1-\zeta^{2}} & \zeta \omega_{n}
\end{array}\right]
$$

Table 2. Feeder controller gains

\begin{tabular}{c|c|c|c|c}
\hline Gain & DE & RL & LQR & LE \\
\hline$K_{p}$ & -6.421 & 25.5085 & - & - \\
$K_{i}$ & $7,340.4$ & $19,417.0$ & 5 & - \\
$K_{d}$ & 0.0963 & 0.3173 & - & - \\
$N$ & $1,640.4$ & $3,174.0$ & - & - \\
$K$ & - & - & {$[0.03920 .0066]$} & {$[0.02320 .0017]$} \\
\hline
\end{tabular}

\subsection{Description of the test environment}

Considering the controllers designed in the previous section, the microgrid simulation is performed in Simulink/MATLAB. The system is tested for small variations in CPL power for the performance analysis of the feeder controllers.

The proposed microgrid is shown in Fig. 4. The simulation starts at time $t=0.0 \mathrm{~s}$. At $t=1.0 \mathrm{~s}$ the feeder runs around the designed operating point and at $t=1.5 \mathrm{~s}$ the feeder's loop is closed for the controller's actuation. Then, the CPL is connected to the system at $t=3.0 \mathrm{~s}$ and it achieves the designed operation point at $t=4.0 \mathrm{~s}$. The CPL controller is connected to the system at $t=4.5 \mathrm{~s}$.

With the system operating around the chosen operating points, the CPL power variation is done. At instants $t=5.0 \mathrm{~s}$ and $t=5.5 \mathrm{~s}$ there are step variations of $0.1 \mathrm{pu}$ in the reference, and the system returns to the original reference value at $t=6.0 \mathrm{~s}$. At $t=6.5 \mathrm{~s}$ and $t=7.0 \mathrm{~s}$, there are other step variations $-0.1 \mathrm{pu}$ in the power reference for the instants $t=7.5 \mathrm{~s}$ and $t=8.0 \mathrm{~s}$ to return in the reference with step variations of $0.1 \mathrm{pu}$. The proposed system analyzes are performed from time $t=5 \mathrm{~s}$, when the controllers are already working and the $\mathrm{CPL}$ is already connected to the DC bus.

\section{RESULTS ANALYSIS}

The microgrid showed in Fig. 4 is tested for small CPL power reference variations. It is seen if the controllers proposed for the feeder can keep the system stable. Also, the performance of each controller is verified through the performance index (PI). Among the indices to be contemplated in this work, integral of square error (ISE) is used to verify the system with smaller error accumulation, shown in Fig. 7a, to maintain the regulated system, also is contemplated the integral of square error weighted by time (ISET), Fig. 7a, to ponder the speed with which the controllers have been able to stabilize the microgrid and finally is verified the integral of the square of the signal of control (ISSC), Fig. 7a, to observe the system energetically better.

\subsection{Performance of controllers designed for the feeder}

With the system at the point of operation, the CPL power reference variation is implemented. The result in time is shown in Fig. 6, wherein Fig. 6a is shown the voltage at which the feeder feeds the DC bus to the power reference variation shown in Fig. 6b.

It is observed by the voltage in the DC bus, Fig. 6a, that the controllers designed for the feeder can keep the system stable even in the occurrence of power variations in the CPL, where the LE methodology showed fewer oscillations about the others metrics.

The performance of controllers is analyzed in terms of IP. In Fig. 7a the ISE for each proposed methodology is 


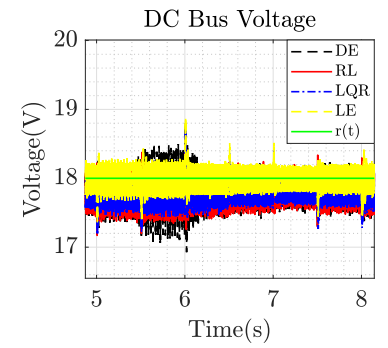

(a)

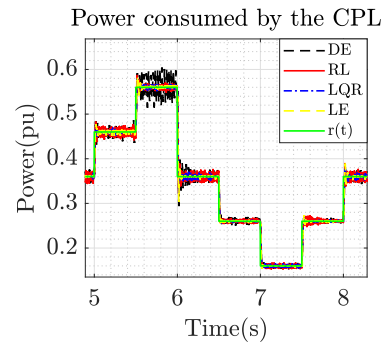

(b)
Fig. 6. (a) Voltage at the output of the feeder for (b) power variation in the CPL and perfomance index for design controller in the feeder.

shown. It is observed that the LE methodology presents the lowest ISE among the four methodologies, indicating better performance in the mitigation of the CPL.

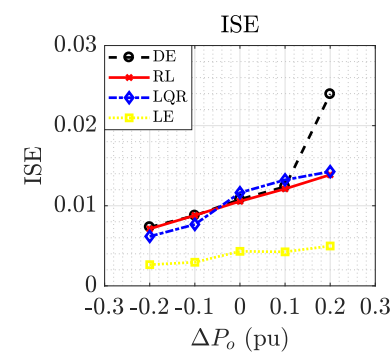

(a)

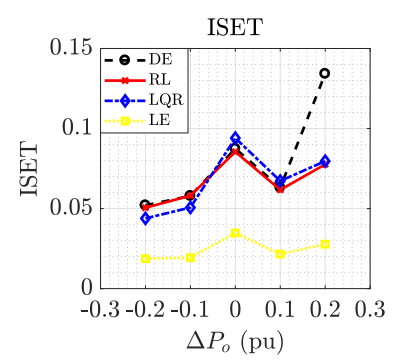

(b)

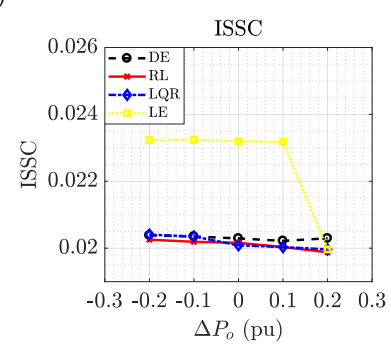

(c)

Fig. 7. (a) integral of square error (ISE) (b) integral of square error weighted by time (ISET) (c) integral of square signal of control (ISSC).

Fig. 7b shows ISET the feeder controllers by considering variations on the load power demand of CPL. The LE methodology is shown with lower ISET among the four methodologies, indicating less time to mitigate the oscillations from the CPL.

Fig. 7c describes the ISSC for the compared control methodologies. In the ISSC the LE methodology presented a higher index about the other methodologies used, indicating that to guarantee the best performance in the mitigation of oscillations, a greater control effort of the system is required. The DE, RL and LQR methodologies presented similar behavior in the three indices, where it is observed that for the increase of the power variation in the CPL the DE methodology has the greater loss of performance and increase in the time of mitigation of the oscillations. The greatest control effort of the controller by LE is related to the duty cycle variation around the operating point. In this case, the LE control design presents a greater duty cycle variation compared to the other methodologies used.

\section{CONCLUSION}

This paper investigates the problem of oscillation damping in DC microgrids. In particular, four control methodologies, namely, DE, RL, LQR, and LE, are used to ensure the stability and oscillation damping in DC microgrids with CPL. Thus, a comparative analysis between that control methodologies is performed. For this purpose, ISE, ISET, and ISSC are evaluated such that the dynamic behavior of microgrid is analyzed to three aspects: damping performance, time spent to mitigate the oscillations, and control effort.

Therefore, it is observed that the LE methodology presents the best performance in the mitigation of the oscillations from the CPL, being able to suppress the oscillations in the shortest time about the other methodologies, as can be seen in the presented ISE and ISET charts. However, it results in a greater effort about other methodologies, as observed in the presented ISSC chart. The other methodologies presented similar performance for mitigation of oscillations, with the observation of the DE that loses performance for excessive variations of power in the CPL.

\section{REFERENCES}

Chen, C.T. (1999). Linear system theory and design. Oxford university press, $3^{\circ}$ edition.

D'Azzo, J.J. and Houpius, C.H. (1988). Linear Control Systems Analysis and Design. McGraw-Hill Education. Elsayed, A.T., Mohamed, A.A., and Mohammed, O.A. (2015). DC microgrids and distribution systems: An overview. Electric Power Systems Research, 119, 407 417.

Erickson, R.W. and Maksimović, D. (2004). Fundamentals of Power Eletronics. Kluwer Academic Publishers, 2nd edition.

Maccari, L.A., Valle, R.L., Ferreira, A., Barbosa, P.G., and Montagner, V. (2016). A LQR design with rejection of disturbances and robustness to load variations applied to a buck converter. Eletrônica de Potência, 21, 7-15.

Marcillo, K.L., Guingla, D.P., Barra, W., de Medeiros, R.L.P., Rocha, E.M., Benavides, D.A.V., and Nogueira, F.G. (2019). Interval robust controller to minimize oscillations effects caused by constant power load in a dc multi-converter buck-buck system. IEEE Access, 7, 26324-26342.

Mosskull, H. (2018). Constant power load stabilization. Control Engineering Practice, 72, 114 - 124.

Poodeh, M.B., Eshtehardiha, S., and Zare, M. (2007). Application of fuzzy logic to control the dc-dc converter. 34-39.

Riccobono, A. and Santi, E. (2014). Comprehensive review of stability criteria for DC power distribution systems. IEEE Transactions on Industry Applications, 50(5), 3525-3535.

Singh, S., Gautam, A.R., and Fulwani, D. (2017). Constant power loads and their effects in DC distributed power systems: A review. Renewable and Sustainable Energy Reviews, 72, $407-421$. 